\title{
DOUBLE COVERS AND CALABI-YAU VARIETIES
}

\author{
SŁAWOMIR CYNK and TOMASZ SZEMBERG \\ Instytut Matematyki, Uniwersytet Jagielloński \\ Reymonta 4, PL-30-059 Kraków, Poland \\ E-mail: cynk@im.uj.edu.pl,szemberg@im.uj.edu.pl
}

Introduction. In the present paper we study examples of double coverings of the projective space $\mathbb{P}^{3}$ branched over an octic surface. A double covering of $\mathbb{P}^{3}$ branched over a smooth octic is a Calabi-Yau threefold. If the octic is singular then so is the double covering and we study its resolution of singularities. In this paper we restrict our considerations to the case of octics with only non-isolated singularities of a special type, namely looking locally like plane arrangements.

Our research was inspired by a paper of Persson [5] where K3 surfaces arising as double covers of $\mathbb{P}^{2}$ branched over curves of degree six are studied. In this note we also adopt some methods introduced in [3] by Hunt in studying Fermat covers of $\mathbb{P}^{3}$ branched over plane arrangements.

The main results of this note are Theorem 2.1 and Theorem 3.5 which can be formulated together as follows

THEOREM. Let $S \subset \mathbb{P}^{3}$ be an octic arrangement with no $q$-fold curve for $q \geq 4$ and no $p$-fold point for $p \geq 6$. Then the double covering of $\mathbb{P}^{3}$ branched along $S$ has a non-singular model $Y$ which is a Calabi-Yau threefold.

Moreover if $S$ contains no triple elliptic curves and $l_{3}$ triple lines then the Euler characteristic $e(Y)$ of $Y$ is given as follows

$$
\begin{aligned}
e(Y)=8-\sum_{i}\left(d_{i}^{3}-4 d_{i}^{2}\right. & \left.+6 d_{i}\right)+2 \sum_{i \neq j}\left(4-d_{i}-d_{j}\right) d_{i} d_{j} \\
& -\sum_{i \neq j \neq k \neq i} d_{i} d_{j} d_{k}+4 p_{4}^{0}+3 p_{4}^{1}+16 p_{5}^{0}+18 p_{5}^{1}+20 p_{5}^{2}+l_{3},
\end{aligned}
$$

where $d_{i}$ denotes the degree of the arrangement surfaces and $p_{i}^{j}$ the number of $i$-fold points contained in $j$ triple curves.

Both authors were partially supported by KBN grant 2 P03A 08310 . They are fellows of the Foundation for Polish Science.

1991 Mathematics Subject Classification: 14E25.

Received by the editors: December 20, 1996; in the revised form: May 30, 1997.

The paper is in final form and no version of it will be published elsewhere. 
For the arrangements we allow exactly six types of singularities. For each case we describe precisely the resolution of singularities in the double cover. Then we study the effect on the Euler number of every blowing-up. This leads to the formula on the Euler number of $Y$ as stated in the above Theorem. Using this formula we obtain a table of examples of Calabi-Yau threefolds with 63 different Euler numbers. In the view of the mirror symmetry it is important to have examples of Calabi-Yau threefolds with as many Euler numbers as possible (it is conjectured that there are only finitely many possible numbers to appear as the Euler number of a Calabi-Yau threefold).

1. Admissible blowing-ups. Let $D$ be a reduced divisor on a smooth threefold $X$. We assume that $D$ is even as an element of the Picard group Pic $X$, this means that there exists $B \in \operatorname{Pic} X$ such that $D \cong 2 B$. In this case there exists a double covering $\pi: Y \rightarrow X$ branched along $D$. If the divisor is smooth then $Y$ is also smooth and we have

$$
\begin{aligned}
& h^{i}\left(\mathcal{O}_{Y}\right)=h^{i}\left(\mathcal{O}_{X}\right)+h^{i}\left(\mathcal{O}_{X}(-B)\right), \\
& K_{Y} \cong \pi^{*}\left(K_{X}+B\right) \\
& e(Y)=2 e(X)-e(D) .
\end{aligned}
$$

If $D$ is singular then the type of its singularities determines singularities of $Y$. Hence it is enough to consider an embedded resolution $D^{\prime}$ of $D$ to obtain a resolution for $Y$. The problem however is to ensure that the resulting divisor is even.

Persson, studying double sextics, introduced in [5] a notion of inessential singularities, i.e. such which do not affect the Euler characteristic and the canonical divisor of the double cover. In a threedimensional case an analogous characterization would be much more complicated. Moreover in our paper we are interested in Calabi-Yau manifolds with many different Euler numbers. This leads to the following description of blowing-ups which do not affect the first Betti number and the canonical divisor of the double cover.

Let $X$ be a smooth threefold and $D \subset X$ an even, reduced divisor. Let $Z \subset D$ be a smooth irreducible proper subvariety and let $\sigma: \widetilde{X} \rightarrow X$ be the blowing-up of $X$ in $Z$ with an exceptional divisor $E$. By mult $_{Z / D}$ we denote the generic multiplicity of $D$ at $Z$ and by $\widetilde{D}$ the proper transform of $D$. Then the divisor $D^{*} \subset \widetilde{X}$ defined as

$$
D^{*}:= \begin{cases}\widetilde{D} & \text { if } \text { mult }_{Z / D} \text { is even, } \\ \widetilde{D}+E & \text { if } \text { mult }_{Z / D} \text { is odd }\end{cases}
$$

is the only reduced and even divisor satisfying $\widetilde{D} \leq D^{*} \leq \sigma^{*} D$.

Definition 1.1. Let $Z \subset D \subset X$ be as above. We call the blowing-up $\sigma: \widetilde{X} \rightarrow X$ admissible iff

$$
K_{\widetilde{X}}+\frac{1}{2} D^{*} \cong \sigma^{*}\left(K_{X}+\frac{1}{2} D\right)
$$

The following proposition gives a characterization of admissible blowing-ups.

Proposition 1.2. On a smooth threefold $X$ there are exactly four types of admissible blow-ups:

C) blowing-up of a curve $Z$ with mult $_{Z / D}$ equal to 2 or 3 ,

$\mathrm{P})$ blowing-up of a point $Z$ with mult $_{Z / D}$ equal to 4 or 5 . 
Proof. Let $r$ denote the codimension of $Z$ in $X$ and let $m=$ mult $_{Z / D}$. Furthermore let $\varepsilon$ be equal 0 if $m$ is even and 1 otherwise. Then we have $K_{\widetilde{X}} \cong \sigma^{*} K_{X}+(r-1) E$ and $D^{*}=\sigma^{*} D-(m-\varepsilon) E$. Therefore

$$
K_{\widetilde{X}}+\frac{1}{2} D^{*} \cong \sigma^{*}\left(K_{X}+D\right)+\left(r-1-\frac{m-\varepsilon}{2}\right) E .
$$

It follows that $\sigma$ is admissible if and only if $m=2(r-1)+\varepsilon$. As the only solutions for $r=2$ we get $m=2$ or $m=3$ and for $r=3$ respectively $m=4$ or $m=5$.

2. Resolution of singularities of the branch locus. Before we start to resolve singularities we need some definitions. We consider octic surfaces in $S \subset \mathbb{P}^{3}$ which locally look like an arrangement of planes (see [3, p. 109]). More precisely, let $S$ be the sum of smooth irreducible surfaces $S_{1}, \ldots, S_{r}$ contained in a smooth threefold $U$. We suppose that

1. For any $i \neq j$ the surfaces $S_{i}$ and $S_{j}$ intersect transversally along a smooth irreducible curve $C_{i, j}$ or they are disjoint.

2. The curves $C_{i, j}, C_{k, l}$ either coincide or intersect transversally.

We call a surface satisfying the above conditions an arrangement. If $U=\mathbb{P}^{3}$ and surfaces $S_{1}, \ldots, S_{r}$ are of degree $d_{1}, \ldots, d_{r}$ respectively, with $d_{1}+\ldots+d_{r}=8$, then we call $S$ an octic arrangement.

We say that an irreducible curve $C \subset S$ is a $q$-fold curve if exactly $q$ of surfaces $S_{1}, \ldots, S_{r}$ pass through it. A point $P \in S$ is called a $p$-fold point if exactly $p$ of the surfaces $S_{1}, \ldots, S_{r}$ pass through it.

THEOREM 2.1. Let $S \subset \mathbb{P}^{3}$ be an octic arrangement with no q-fold curve for $q \geq 4$ and no $p$-fold point for $p \geq 6$. Then there exists a sequence $\sigma=\sigma_{1} \circ \ldots \circ \sigma_{s}: \mathbb{P}_{3}^{*} \rightarrow \mathbb{P}^{3}$ of admissible blowing-ups and a smooth and even divisor $S^{*} \subset \mathbb{P}_{3}^{*}$ such that $\sigma_{*}\left(S^{*}\right)=S$ and the double covering $Y$ of $\mathbb{P}_{3}^{*}$ branched over $S^{*}$ is a smooth Calabi-Yau threefold.

Proof. The proof consists of careful resolving the singularities of $S$.

a) Fivefold points. First we blow up $\sigma_{1}: \mathbb{P}_{(1)}^{3} \rightarrow \mathbb{P}^{3}$ all points $P \in \mathbb{P}^{3}$ which are 5-fold points of $S$. Then in the exceptional divisor $\mathbb{P}^{2}$ over each of these points we get one of the following configurations of lines - exceptional divisors for surfaces passing through $P$.

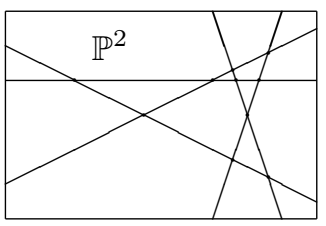

Case a-1

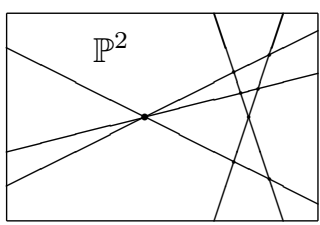

Case a-2

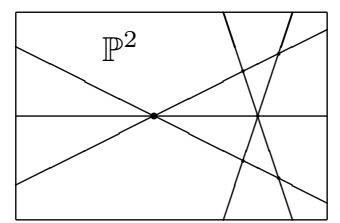

Case a-3

The obtained cases depend on the number of threefold curves passing through $P$. Now we replace $S$ by its strict transform plus the exceptional divisor, and we call this new branch locus $S_{1}$. If there were no 5 -fold points on $S$ we have $S_{1}=S$. We observe that $S_{1}$ contains no 5 -fold points. 
b) Triple curves. If there are triple curves on $S_{1}$ we blow them up $\sigma_{2}: \mathbb{P}_{(2)}^{3} \rightarrow \mathbb{P}_{(1)}^{3}$. Let $C \subset S_{1}$ be a triple curve. Then in the exceptional divisor $C \times \mathbb{P}^{1}$ we get the following configuration where $C_{1}, C_{2}, C_{3}$ are isomorphic to $C$ and $L_{1}, \ldots, L_{t}$ are lines, with $t$ equal to the number of 4-fold points on $C$.

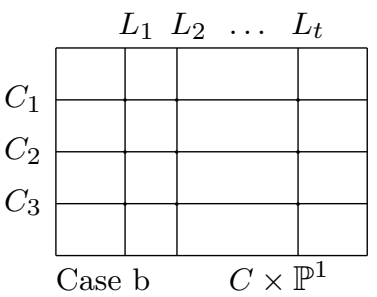

As a new branch locus for the double cover we take $S_{2}$ equal to the strict transform of $S_{1}$ plus the exceptional divisors. There are no 5 -fold points and no triple curves on $S_{2}$.

c) Fourfold points. Now we blow up $\sigma_{3}: \mathbb{P}_{(3)}^{3} \rightarrow \mathbb{P}_{(2)}^{3}$ all the 4 -fold points of $S_{2}$. In the exceptional divisor over a 4-fold point $P \in S_{2}$ we get the following configuration.

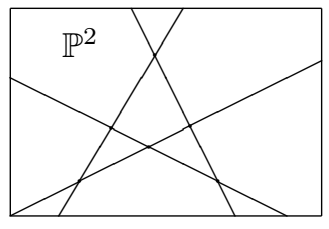

Case c

Now as $S_{3}$ we take the strict transform of $S_{2}$. On $S_{3}$ there are no more singularities of types a), b), c).

d) Double curves. In the last step we blow up $\sigma_{4}: \mathbb{P}_{3}^{*}=$ $\mathbb{P}_{(4)}^{3} \rightarrow \mathbb{P}_{(3)}^{3}$ the double curves on $S_{3}$ and take $S^{*}=S_{4}$ as the strict transform of $S_{3}$. Since $S$ was an octic arrangement we get $S^{*}$ smooth and even as an element of Pic $\mathbb{P}_{3}^{*}$.

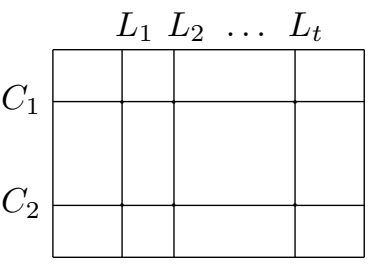

ruled surface over $C$ Case d

Let $\sigma=\sigma_{4} \circ \ldots \circ \sigma_{1}$ and let $\pi: Y \rightarrow \mathbb{P}_{3}^{*}$ be the double covering branched over $S^{*}$. Using the adjunction formula and the Serre duality we get

$$
K_{Y} \cong \pi^{*}\left(K_{\mathbb{P}_{3}^{*}}+\frac{1}{2} S^{*}\right) \cong \pi^{*}\left(\sigma^{*}\left(K_{\mathbb{P}^{3}}+\frac{1}{2} S\right)\right)=\mathcal{O}_{Y}
$$

and

$$
\begin{aligned}
h^{1}\left(\mathcal{O}_{Y}\right)=h^{1}\left(\mathcal{O}_{\mathbb{P}_{3}^{*}}\right)+h^{1}\left(\mathcal{O}_{\mathbb{P}_{3}^{*}}\left(-\frac{1}{2} S^{*}\right)\right) \\
\quad=h^{1}\left(\mathcal{O}_{\mathbb{P}_{3}^{*}}\right)+h^{2}\left(\mathcal{O}_{\mathbb{P}_{3}^{*}}\left(K_{\mathbb{P}_{3}^{*}}+\frac{1}{2} S^{*}\right)\right)=h^{1}\left(\mathcal{O}_{\mathbb{P}^{3}}\right)+h^{2}\left(\mathcal{O}_{\mathbb{P}^{3}}\right)=0 .
\end{aligned}
$$

R e m a r k 2.2. Observe that the first three steps of the above resolution are uniquely determined whereas the last step is defined only upto the order in which we blow up the double curves. Change in the order in which the double curves are blown up may lead to a flop of the resulting threefold, see e.g. [4]. However this does not affect the Euler number in which we are interested. Hence we do not distinguish between birational models differing by a flop.

3. Euler characteristic of double octics. In this section we compute the Euler characteristic of Calabi-Yau threefolds obtained from octic arrangements as in Theorem 2.1.

For an arrangement $S$ we introduce the following notation: 
$e^{*}(S)$ is the sum of Euler numbers of all components of $S$,

$E_{p}(S)$ is the sum of Euler numbers of $p$-fold curves on $S$,

$p_{j}(S)$ is the number of isolated $j$-fold points on $S$,

$p_{j}^{k}(S)$ is the number of isolated $j$-fold points lying on exactly $k$ triple curves.

Now we compute how the above data changes under blowing-ups described in the proof of Theorem 2.1 .

Proposition 3.1. Let $S \subset U$ be an arrangement in a threefold $U$. Let $\sigma: V \rightarrow U$ be a blowing-up of the type a), b), c) or d) with the center $Z$ and the exceptional divisor $E$. As before $S^{*}=\sigma^{*} S+\varepsilon E$, with $\varepsilon=0$ or 1 depending on the case $\left.\mathrm{a}-\mathrm{d}\right)$. In this situation we have

$$
\begin{aligned}
& 2 e(U)-e^{*}(S)+2 E_{2}(S)-p_{3}(S)+6 E_{3}(S)+12 p_{5}^{2}(S)+9 p_{5}^{1}(S)+6 p_{5}^{0}(S) \\
& =2 e(V)-e^{*}\left(S^{*}\right)+2 E_{2}\left(S^{*}\right)-p_{3}\left(S^{*}\right)+6 E_{3}\left(S^{*}\right)+12 p_{5}^{2}\left(S^{*}\right)+9 p_{5}^{1}\left(S^{*}\right)+6 p_{5}^{0}\left(S^{*}\right) .
\end{aligned}
$$

P r o of. The following table describes how the blowing-up affects the Euler numbers of the threefold and the arrangement. The next table shows changes of the combinatorial data. If $Z$ is a $q$-fold line then $t$ denotes the number of $(q+1)$-fold points on $Z$.

\begin{tabular}{|c|c|c|c|c|}
\hline type & $e(V)-e(U)$ & $e^{*}\left(S^{*}\right)-e^{*}(S)$ & $E_{2}\left(S^{*}\right)-E_{2}(S)$ & $E_{3}\left(S^{*}\right)-E_{3}(S)$ \\
\hline $\mathrm{a}-1$ & 2 & 8 & 10 & 0 \\
\hline $\mathrm{a}-2$ & 2 & 8 & 10 & 0 \\
\hline $\mathrm{a}-3$ & 2 & 8 & 10 & 0 \\
\hline $\mathrm{b}$ & $e(Z)$ & $2 e(Z)+t$ & $3 e(Z)+2 t$ & $-e(Z)$ \\
\hline $\mathrm{c}$ & 2 & 4 & 0 & 0 \\
\hline $\mathrm{d}$ & $e(Z)$ & $t$ & $-e(Z)$ & 0 \\
\hline
\end{tabular}

\begin{tabular}{|c|c|c|c|c|}
\hline type & $p_{3}\left(S^{*}\right)-p_{3}(S)$ & $p_{5}^{0}\left(S^{*}\right)-p_{5}^{0}(S)$ & $p_{5}^{1}\left(S^{*}\right)-p_{5}^{1}(S)$ & $p_{5}^{2}\left(S^{*}\right)-p_{5}^{2}(S)$ \\
\hline a-1 & 10 & -1 & 0 & 0 \\
\hline a-2 & 7 & 0 & -1 & 0 \\
\hline a-3 & 4 & 0 & 0 & -1 \\
\hline b & $3 t$ & 0 & 0 & 0 \\
\hline c & 0 & 0 & 0 & 0 \\
\hline d & $-t$ & 0 & 0 & 0 \\
\hline
\end{tabular}

It is simple to check the entries of the above tables and then case by case to verify that the term given in the proposition remains invariant.

As a corollary from the above proposition we obtain the following

Proposition 3.2. In the setup of Theorem 2.1 we have

$$
e(Y)=8-e^{*}(S)+2 E_{2}(S)-p_{3}^{0}(S)+6 E_{3}(S)+12 p_{5}^{2}(S)+9 p_{5}^{1}(S)+6 p_{5}^{0}(S) .
$$

Now we give some formula to compute the invariants used in the above proposition. 
LEMMA 3.3. For an octic arrangement $S$ in $\mathbb{P}^{3}$ we have

and

$$
\begin{gathered}
e^{*}(S)=\sum d_{i}^{3}-4 d_{i}^{2}+6 d_{i}, \\
2 E_{2}(S)+6 E_{3}(S)=2-\sum_{i \neq j}\left(4-d_{i}-d_{j}\right) d_{i} d_{j} .
\end{gathered}
$$

Proof. From the adjunction formula we have

$$
e(X)=d^{3}-4 d^{2}+6 d
$$

for a smooth surface $X \subset \mathbb{P}^{3}$ of degree $d$. Similarly if $C \subset \mathbb{P}^{3}$ is a smooth complete intersection of surfaces of degree $d_{1}, d_{2}$ we have

$$
e(C)=\left(4-d_{1}-d_{2}\right) d_{1} d_{2} .
$$

In [3] a formula for the number of singular points of an arrangement of planes is given. This formula can be generalized to the case of an arbitrary octic arrangement. In the simplest case if there are no triple curves we have

$$
\sum_{q \geq 3}\left(\begin{array}{l}
q \\
3
\end{array}\right) p_{q}=\sum_{i \neq j \neq k \neq i} d_{i} d_{j} d_{k} .
$$

Situation becomes more complicated if there are also triple curves in the arrangement. Since $\operatorname{deg}(S)=8$ there are only two possibilities:

- either there is one triple elliptic curve and no more triple curves

- or there are only triple lines.

We can easily classify arrangements with a triple elliptic curve

- $d_{1}=d_{2}=d_{3}=d_{4}=2, \quad p_{3}=8, \quad p_{5}=0, \quad e=-16$;

- $d_{1}=d_{2}=d_{3}=2, \quad d_{4}=d_{5}=1, \quad p_{3}=6, \quad p_{5}=0, \quad e=12$

- $d_{1}=d_{2}=d_{3}=2, \quad d_{4}=d_{5}=1, \quad p_{3}=3, \quad p_{5}^{0}=p_{5}^{2}=0, \quad p_{5}^{1}=1, \quad e=24 ;$

- $d_{1}=d_{2}=d_{3}=2, \quad d_{4}=d_{5}=1, \quad p_{3}=0, \quad p_{5}^{0}=p_{5}^{2}=0, \quad e=36$;

LEMMA 3.4. For an arrangement with $l_{3}$ triple lines $\left(l_{3}=\frac{1}{2} E_{3}\right)$ and no triple elliptic curves we get the following formulas:

$$
\begin{gathered}
p_{3}+4 p_{4}+10 p_{5}-\left(p_{4}^{1}+p_{5}^{1}+2 p_{5}^{2}-l_{3}\right)=\sum_{i \neq j \neq k \neq i} d_{i} d_{j} d_{k}, \\
5 l_{3}=p_{4}^{1}+2 p_{5}^{1}+4 p_{5}^{2} .
\end{gathered}
$$

Proof. The number on the right-hand side of the first equation is just the sum of intersection numbers of all possible triples of arrangement surfaces, i.e. the number of triple points in case all intersections are transversal and reduced. On the left-hand side we take account of the multiple points and multiple lines. For example, if there is a triple line in the picture then it is an intersection of three planes (cf. condition 1 for the arrangement) and corresponds to one "lost" point. A 4-fold point corresponds to 4 "lost" points etc.

The second formula follows from the Bézout theorem applied to all triple lines in the arrangement. Such a line lies on three planes and intersects the remaining quintic properly.

From Proposition 3.2, Lemma 3.3 and Lemma 3.4 we get the main result of this paper. 
THEOREM 3.5. If $S$ is an octic arrangement with no triple elliptic curve then

$$
\begin{aligned}
e(Y)=8-\sum_{i}\left(d_{i}^{3}-4 d_{i}^{2}\right. & \left.+6 d_{i}\right)+2 \sum_{i \neq j}\left(4-d_{i}-d_{j}\right) d_{i} d_{j} \\
& -\sum_{i \neq j \neq k \neq i} d_{i} d_{j} d_{k}+4 p_{4}^{0}+3 p_{4}^{1}+16 p_{5}^{0}+18 p_{5}^{1}+20 p_{5}^{2}+l_{3} .
\end{aligned}
$$

4. Examples. In this section we apply the preceding results to various examples of octic arrangements and for the double coverings we get 63 distinct Euler numbers. We collect the data describing an arrangement and the corresponding Euler number. Some Euler numbers arise from more than one arrangement, the table contains only one

\begin{tabular}{|c|c|c|c|c|c|c|c|}
\hline$\left(d_{1}, \ldots, d_{r}\right)$ & $p_{4}{ }^{0}$ & $p_{4}{ }^{1}$ & $p_{5}{ }^{0}$ & $p_{5}{ }^{1}$ & $p_{5}{ }^{2}$ & $l_{3}$ & $\mathbf{e}(\mathbf{Y})$ \\
\hline 8 & & & & & & & -296 \\
\hline 1,7 & & & & & & & -240 \\
\hline 2,6 & & & & & & & -200 \\
\hline $1,1,6$ & & & & & & & -180 \\
\hline 3,5 & & & & & & & -176 \\
\hline 4,4 & & & & & & & -168 \\
\hline $1,2,5$ & & & & & & & -140 \\
\hline \multirow{2}{*}{$1,1,1,5$} & & & & & & & -120 \\
\hline & 1 & & & & & & -116 \\
\hline $2,2,4$ & & & & & & & -104 \\
\hline $2,3,3$ & & & & & & & -92 \\
\hline \multirow[t]{3}{*}{$1,1,2,4$} & & & & & & & -84 \\
\hline & 1 & & & & & & -80 \\
\hline & 2 & & & & & & -76 \\
\hline \multirow[t]{2}{*}{$1,1,3,3$} & & & & & & & -72 \\
\hline & 1 & & & & & & -68 \\
\hline \multirow[t]{2}{*}{$1,1,1,1,4$} & & & & & & & -64 \\
\hline & 1 & & & & & & -60 \\
\hline \multirow[t]{2}{*}{$1,2,2,3$} & & & & & & & -56 \\
\hline & 1 & & & & & & -52 \\
\hline $1,1,1,1,4$ & & & 1 & & & & -48 \\
\hline $1,2,2,3$ & 3 & & & & & & -44 \\
\hline $2,2,2,2$ & & & & & & & -40 \\
\hline \multirow[t]{4}{*}{$1,1,1,2,3$} & & & & & & & -36 \\
\hline & 1 & & & & & & -32 \\
\hline & 2 & & & & & & -28 \\
\hline & 3 & & & & & & -24 \\
\hline $1,1,2,2,2$ & & & & & & & -20 \\
\hline \multirow[t]{4}{*}{$1,1,1,1,1,3$} & & & & & & & -16 \\
\hline & 1 & & & & & & -12 \\
\hline & 2 & & & & & & -8 \\
\hline & 3 & & & & & & -4 \\
\hline
\end{tabular}
example in order to keep it reasonable short. 


\begin{tabular}{|c|c|c|c|c|c|c|c|}
\hline$\left(d_{1}, \ldots, d_{r}\right)$ & $p_{4}{ }^{0}$ & $p_{4}{ }^{1}$ & $p_{5}{ }^{0}$ & $p_{5}{ }^{1}$ & $p_{5}{ }^{2}$ & $l_{3}$ & $\mathbf{e}(\mathbf{Y})$ \\
\hline \multirow[t]{5}{*}{$1,1,1,1,2,2$} & & & & & & & 0 \\
\hline & 1 & & & & & & 4 \\
\hline & 2 & & & & & & 8 \\
\hline & 3 & & & & & & 12 \\
\hline & & & 1 & & & & 16 \\
\hline \multirow[t]{5}{*}{$1,1,1,1,1,1,2$} & & & & & & & 20 \\
\hline & 1 & & & & & & 24 \\
\hline & 2 & & & & & & 28 \\
\hline & 3 & & & & & & 32 \\
\hline & & & 1 & & & & 36 \\
\hline \multirow[t]{21}{*}{$1,1,1,1,1,1,1,1$} & & & & & & & 40 \\
\hline & 1 & & & & & & 44 \\
\hline & 2 & & & & & & 48 \\
\hline & 3 & & & & & & 52 \\
\hline & 4 & & & & & & 56 \\
\hline & 5 & & & & & & 60 \\
\hline & 6 & & & & & & 64 \\
\hline & 7 & & & & & & 68 \\
\hline & 8 & & & & & & 72 \\
\hline & 9 & & & & & & 76 \\
\hline & & 1 & & 2 & & 1 & 80 \\
\hline & & 8 & & 1 & & 2 & 84 \\
\hline & 12 & & & & & & 88 \\
\hline & & 4 & & 1 & 1 & 2 & 92 \\
\hline & & 6 & & 2 & & 2 & 96 \\
\hline & & 7 & & & 2 & 3 & 104 \\
\hline & & 9 & & 1 & 1 & 3 & 108 \\
\hline & & 3 & & & 3 & 3 & 112 \\
\hline & 1 & 3 & & & 3 & 3 & 116 \\
\hline & 2 & 3 & & & 3 & 3 & 120 \\
\hline & & 4 & & & 4 & 4 & 136 \\
\hline
\end{tabular}

Most of arrangements from the above table are easy to construct. We conclude this paper by giving hints as to construct those which, at least to the authors, seem less obvious.

$$
e=-44
$$

We take two general quadrics and a plane. They have four common points. Now, we add a cubic passing through three of these points. The Bertini theorem implies that the transversality conditions are satisfied away of the fourth point. But there they are obviously fulfilled.

$$
e=-24
$$

We take two general planes and a cubic. They have three common points. Then we take a general quadric through two of these points and a plane through the third one. 
$e=52,56,60,64,68,72,76$

We take six planes as the faces of the cube. Then we let other two planes pass through $0,1,2,3,4,5,6$ vertices, respectively.

$e=88$

Here we consider faces of the octahedron. They have four 4 -fold points in the affine part and eight more at the infinity.

$e=104$

We take four planes $P_{1}, P_{2}, P_{3}, P_{4}$ in the general position. Let $P_{5}$ be a general plane through the line $P_{1} \cap P_{2}$ and similarly $P_{6}, P_{7}$ general planes through the lines $P_{2} \cap P_{3}$ and $P_{3} \cap P_{4}$. The eighth plane can be taken generally.

$e=112,116,120$

We consider two tetrahedra glued by a face and take their seven faces. The two vertices not lying on the common face are triple points. Then we add a plane through none, one or two of them.

$e=136$

We take four planes $P_{1}, P_{2}, P_{3}, P_{4}$ in the general position and add a general plane through each of the lines $P_{1} \cap P_{2}, P_{1} \cap P_{3}, P_{2} \cap P_{4}, P_{3} \cap P_{4}$.

Finally, we remark that many more examples of Euler numbers can be obtained allowing additionally to the non-isolated singularities some isolated ones, e.g. double points. Similarly one can weaken the assumption on the reducibility of the arrangement allowing surfaces with selfintersections. These aspects will be studied elsewhere.

Acknowledgments. The authors would like to thank the Referee for helpful remarks.

\section{References}

[1] J. Harris, Algebraic Geometry. A First Course, Graduate Texts in Math. 133, Springer, New York, 1992.

[2] R. Hartshorne, Algebraic Geometry, Graduate Texts in Math. 52, Springer, New York, 1977.

[3] B. Hunt, Complex manifold geography in dimension 2 and 3, J. Differential Geom. 30 (1989), $51-153$.

[4] J. Kollár, Flops, Nagoya Math. J. 113 (1989), 15-36.

[5] U. Persson, Double sextics and singular K3 surfaces, in: Algebraic Geometry, Sitges (Barcelona) 1983, Lecture Notes in Math. 1124, Springer, Berlin, 1985, 262-328. 